\title{
Erratum to: Current strategy for triple-negative breast cancer: appropriate combination of surgery, radiation, and chemotherapy
}

\author{
Hiroshi Yagata $\cdot$ Yuka Kajiura $\cdot$ Hideko Yamauchi
}

Published online: 14 July 2012

(C) The Japanese Breast Cancer Society 2012

\section{Erratum to: Breast Cancer (2011) 18:165-173 \\ DOI 10.1007/s12282-011-0254-9}

In the article cited above, in the "Systemic therapy" section, under the subheading " $F E C-D T X$," the third paragraph appeared incorrectly. The correct paragraph is as follows:

In 13 patients (22.4\%), tumors were unchanged or grew slightly in response to FEC. Distant metastases developed in $7(53.8 \%)$ of these patients, resulting in death. In only 1 patient, the tumor shrank dramatically and reached a pCR after treatment with DTX. The patient is alive without any recurrence. The tumor increased in diameter, but central necrosis developed in response to FEC, suggesting that FEC was effective (Fig. 3). After shrinkage in response to FEC, tumors grew during treatment with DTX in 9 patients $(15.5 \%)$. Tumor growth was detected in the third cycle of DTX in only 1 patient, and treatment was withdrawn. In the other 8 patients, tumor growth was not detected until the completion of 4 cycles of DTX. These patients thus received ineffective, unnecessary treatment. Our results indicate that even patients who have tumor shrinkage in response to FEC should be closely followed up during subsequent treatment with DTX.

The online version of the original article can be found under doi: 10.1007/s12282-011-0254-9.

H. Yagata $(\bowtie) \cdot$ Y. Kajiura $\cdot$ H. Yamauchi Department of Breast Surgical Oncology,

St. Luke's International Hospital, 9-1 Akashi-cho,

Chuo-ku, Tokyo 104-8560, Japan

e-mail: yagata@mvh.biglobe.ne.jp 